\title{
Determination of the strain-energy release rate of a composite laminate under high- rate tensile deformation in fibre direction
}

Justus Hoffmann ${ }^{\mathrm{a},}{ }^{*}$, Hao Cuib ${ }^{\mathrm{b}}$ Nik Petrinic ${ }^{\mathrm{a}}$

aDepartment of Engineering Science, University of Oxford, Parks Road, Oxford, OX1 3PJ, UK

${ }^{b}$ Centre for Aeronautics, Cranfield University, UK

*Corresponding author: justus.hoffmann@eng.ox.ac.uk

\section{Abstract}

In order to successfully model design-critical impact loading events on laminated composite structures, the rate-dependency of the composite material has to be correctly reflected. In this context, the rate-dependency of the strain-energy release rate for fibre tensile failure under high-rate loading conditions has not yet been satisfyingly explored. This study employed compact tension specimens consisting of IM7/8552 for dynamic testing on a split-Hopkinson tension bar system. Data reduction was based on the area method. The obtained strain-energy release rate for testing under high-rate conditions was determined to $G_{I c, \text { dyn }}^{f+}=82.0 \pm 20.8 \mathrm{~kJ} / \mathrm{m}^{2}$, exhibiting a salient drop compared to its counterpart obtained under quasi-static loading $\left(G_{I c, Q S}^{f+}=195.8 \pm 18.0 \mathrm{~kJ} / \mathrm{m}^{2}\right)$. Analysis of the strain field surrounding the crack tip using digital image correlation (DIC) suggested a more extensive damage zone for testing under quasi-static than for high-rate loading. A fractographic analysis of the 
specimens did not indicate any pronounced difference in terms of fracture surface morphology across the two loading rate regimes.

Keywords: (A) Polymer-matrix composites (PMCs); (B) Fracture toughness; (D) Fractography; (D) Scanning electron microscopy (SEM); fibre failure

\section{Introduction}

Owing to their extraordinary mechanical properties, composite materials experience increasing usage in high-tech applications such as the aerospace sector (e.g., [1,2]). In order to make effective and economical use of the enormous degrees of freedom that this material class has to offer, the design and dimensioning process is nowadays carried out mainly in silico (e.g., [3]). As many design critical load cases, such as birdstrike (e.g., [4]), involve the impact of an object onto a composite structure, the constitutive model reflecting the composite's behaviour should adequately account for the rate-dependent nature of the material in order to yield realistic simulation results (e.g., [5]). The generation of experimental data for constitutive model calibration of a composite's rate-dependent elastic and strength properties is, in principle, well established (e.g., [6-11]). With respect to a composite's dynamic strain-energy release rates, which, in turn, govern the material's strain-softening domain, delamination has been studied for decades now (e.g., $[12,13])$. By comparison, the dynamic strainenergy release rates associated with fibre failure have only very recently become the subject of scientific study. Considering, however, the already salient discrepancy between the matrix- and the fibre-dominated energy release rates under quasi-static loading $\left(G_{I c, Q S}^{\text {delam }}=0.28 \mathrm{~kJ} / \mathrm{m}^{2}\right.$ [14] vs. $\left.G_{I c, Q S}^{f+}=147.2 \mathrm{~kJ} / \mathrm{m}^{2}[15]\right)$, the consequences 
of accounting for a potentially substantial rate-dependency of the latter on a composite structure's overall ability to dissipate energy under impact loading may prove vital for the design and dimensioning process of composite structures.

Against this background, the only study published to date on the dynamic energy release rate of a laminate under deformation in fibre compressive direction already clearly showed a substantial rate-sensitivity $\left(G_{I c, Q S}^{f-}=101.6 \mathrm{~kJ} / \mathrm{m}^{2}\right.$ vs. $G_{I c, d y n}^{f-}=$ $165.6 \mathrm{~kJ} / \mathrm{m}^{2}$; material: IM7/8552; Kuhn et al. (2017) [16]).

Regarding the dynamic fracture of a laminate in fibre tensile direction, the only research undertaken so far was carried out by McCarroll (2011) [17]. The study employed compact tension specimens composed of $I M 7 / 8552$ for dynamic testing on a servo-hydraulic Instron 4505 machine in conjunction with a lost motion device. As the obtained force measurement using this particular setup exhibited strong oscillations, an alternative data reduction scheme was devised, which facilitated calculating the laminate's energy release rate without requiring the force history as input. This data reduction approach was based on two key elements: (i) Substitution of the unusable force signal with an approximation of the applied force based on the measurement of the compressive strain $\varepsilon_{B}$ in the vicinity of the specimen's back end according to $F(a)=k(a) \cdot \varepsilon_{B}(a)$; the factor $k(a)$ was obtained based on a numerical model of the specimen using cohesive elements to reflect the crack path. (ii) The same model was employed to determine the specimen's compliance $C$ as a function of crack tip position $a$; this relationship was then used to infer $C$ based on the experimentally measured value of $a$. The model employed to obtain both parameters, $k(a)$ and $C(a)$, was based 
on quasi-static material properties. Both parameters were assumed to be reasonably rate-insensitive. Ultimately, experimental measurement of $\varepsilon_{B}$ and $a$ then facilitated determining the laminate's energy release rate according to Eq. (1) (with $t_{\text {lam }}$ as the laminate's thickness).

$$
G_{I c}^{l a m}=\frac{k^{2}(a) \cdot \varepsilon_{B}^{2}(a)}{2 t_{l a m}} \cdot \frac{d C}{d a}
$$

Following this data reduction approach McCarroll found $G_{I c}^{f+}$ to exhibit a slight decrease with increasing loading rate, however, this decrease was still within the range of the experimental scatter of the tests carried out under quasi-static loading.

In light of the difficulties experienced by McCarroll to generate a usable force signal using a servo-hydraulic testing machine, an attempt was made in this study to determine the rate-dependent nature of $G_{I c}^{f+}$ based on experiments employing a splitHopkinson bar (SHB) setup, and subsequent data reduction using the area method.

\section{Specimen and material}

The specimen selected for the present study followed a compact tension (CT) design. The specimen's dimensions, as given in Fig. 1, were chosen to be identical to the ones employed by Pinho et al. (2006) [18].

Figure 1 about here

The panel from which the specimens were manufactured was produced by stacking 34 IM7/8552 unidirectionally-reinforced prepreg layers by hand (lay-up sequence: $\left.\left[(90,0)_{8}, 90\right]_{\text {symm }}\right)$ and subsequently curing this laminate according to Hexcel's 
recommended temperature/pressure cycle [19]. The 34 individual plies added up to a total specimen thickness of approximately $4.3 \mathrm{~mm}$. Potential delaminations as a result of the machining process of the specimens' contour and through-holes were avoided by sandwiching the composite in a metallic fixture. A sectioning blade was employed to machine the $0.4 \mathrm{~mm}$ wide slit. The final notch which was meant to act as the crack tip's starting point was produced using a micro saw (blade thickness: $0.1 \mathrm{~mm}$ ). This final step was motivated by Laffan et al.'s (2011) [15] study in which the authors demonstrated that the notch root's radius for an $1 M 7 / 8552$ laminate should be below $250 \mu \mathrm{m}$ for the energy release rate's initial value not to exhibit an artificial elevation. Following the machining process, a black speckle pattern on a white background was applied in order to allow for a digital image correlation-based (DIC) analysis of the displacement field surrounding the propagating crack tip over the course of the fracture process.

\section{Experimental setup and data acquisition}

\subsection{Quasi-static experiments}

Quasi-static testing was carried out on a screw-driven testing machine (Zwick/Roell Z250). Load application was displacement-controlled at a rate $0.5 \mathrm{~mm} / \mathrm{min}$. Force was measured via the testing rig's $20 \mathrm{kN}$ load cell. Opening displacement was obtained employing a laser extensiometer (EIR LE-05). The samples' deformation and fracture process was recorded using a camera (jAi BM-500GE) in conjunction with a Nikon Micro-NIKKOR $55 \mathrm{~mm}$ lens. Images were taken at $1 \mathrm{fps}$ at a resolution of $1884 \times 1520$ pixels. The experimental setup for quasi-static testing is shown in Fig. $\mathbf{2 a .}$ 


\subsection{High-rate experiments}

Testing under dynamic loading conditions was carried out on a $16 \mathrm{~mm}$ diameter titanium split-Hopkinson tension bar system featuring a crescent-shaped projectile (see [20] for details on the bar system and its dimensions); a sketch of the experimental setup is provided in Fig. 3. In addition to the existing bar supports made of polyamide, brass bearings were installed in the specimen's direct vicinity in order to ensure the absence of any non-negligible transverse motion component of the bars caused by the setup's asymmetric nature. Furthermore, a 'separator' mechanism was introduced to prevent the fractured specimen halves to come in contact again after final failure so as to facilitate a post-mortem analysis of the specimens' fracture surfaces. A close-up view of the mounted specimen for dynamic testing is given in Fig. $2 b$.

Figure 2 about here

Figure 3 about here

The loading pulse featured a linearly rising slope which was generated using a $3 \mathrm{~mm}$ thick neoprene pulse shaper in conjunction with a projectile velocity of $7.5 \mathrm{~m} / \mathrm{s}$. The loading pulse's profile and its ability to generate valid testing conditions are discussed in more detail in Sec. 5.2.

Force was measured based on the readings of the strain-gauges affixed to the bars and time-shifted according to one-dimensional wave analysis. In order to accurately measure the specimens' opening displacement, two additional L-shaped sheet metal pieces were mounted to the bar/specimen connector such that they roughly shared a 
common focal plane with the specimen. The purpose of these additional pieces was to transfer the bars' movement into the camera's field of vision, thus allowing for an opening displacement determination via DIC. A depiction of this 'displacement tracker' is given in Fig. 4, alongside a comparison between an opening displacement measurement based on the conventional SHB analysis versus a DIC-based approach, highlighting the necessity to employ the latter method in order to bypass the considerable additional compliance of the loading train that is not accounted for by the conventional SHB analysis. The samples' deformation and fracture process was recorded using a high-speed camera (Specialised Imaging Ltd., model: Kirana) in conjunction with a Nikon Micro-NIKKOR $55 \mathrm{~mm}$ lens. Images were taken at $300,000 \mathrm{fps}$ at a resolution of $924 \times 768$ pixels.

Figure 4 about here

\section{Outline of data reduction process}

\subsection{Determination of $G_{I c}^{\text {lam }}$}

Data reduction was based on the area method. In contrast to other approaches, such as ASTM E399 [21], an FE-based [18] or a DIC-based [22] evaluation of the J-integral, or McCarroll's data reduction scheme [17], data reduction via the area method does not require the composite material's elastic properties as input or for calibration. As composites have been shown to exhibit pronounced rate-dependency for the vast majority of their elastic properties (e.g., [6-10]), employing a data reduction scheme that does not depend on those properties rules out a priori any potentially 
unaccounted influence of these rate-dependent moduli on the calculated energy release rate value under high-rate loading conditions. The force history used for the data reduction process was the arithmetic average of the bar forces $F_{\text {in }}$ and $F_{\text {out }}$ determined based on the classical SHB analysis (e.g., [23]).

Obtaining $G_{I c}^{\text {lam }}$ using the area method is based on relating the dissipated mechanical energy $\Delta U$ to the newly generated free surface area due to crack tip advancement $\Delta a$. An illustration of the area method for 'stick-slip' crack propagation is given in Fig. $\mathbf{5}$. For the case of linear elastic material behaviour, $G_{I c}^{\text {lam }}$ can be obtained as follows [24]:

$$
G_{I c}^{l a m}=\frac{1}{2 t_{l a m} \Delta a}\left(F_{1} x_{2}-F_{2} x_{1}\right)
$$

For the present study, $G_{I c}^{\text {lam }}$ was determined as an averaged value over a series of $N$ crack tip advancements $\Delta a$ according to [25]:

$$
G_{I c}^{l a m}=\frac{1}{2 t_{l a m} N \Delta a} \sum_{i=1}^{N}\left(F_{1 i} x_{2 i}-F_{2 i} x_{1 i}\right)
$$

The motivation for calculating $G_{I c}^{l a m}$ in an averaged sense was to mitigate the effects of: (i) the varying degree of force disequilibrium over the course of crack propagation (see Sec. 5.2), (ii) the inaccuracies introduced by optically determining the crack tip's position (see Laffan et al. (2010) [26]), and (iii) the inaccuracies connected with the comparatively coarse resolution of the crack tip position history caused by the limited number of high-speed camera frames covering the fracture process. 
Figure 5 about here

\subsection{Determination of $G_{I c}^{f+}$}

In order to calculate $G_{I C}^{f+}$ on the basis of the obtained value for $G_{I c}^{\text {lam }}$, Pinho et al.

(2006) [18] proposed Eq. (4) based on two assumptions: (i) the absence of any

substantial interaction between the $0^{\circ}$ and the $90^{\circ}$ plies, and (ii) the negligibility of any other energy dissipation mechanisms.

$$
G_{I c}^{f+}=\frac{t_{l a m}}{t_{0}} G_{I c}^{l a m}-\frac{t_{90}}{t_{0}} G_{I c}^{I F F}
$$

In the above equation, $t_{0}$ denotes the cumulative thickness of all $0^{\circ}$ plies in the laminate, and $t_{90}$ that of all $90^{\circ}$ plies, respectively.

As, however, the energy released by inter-fibre failure (IFF) $G_{I C}^{I F F}$ is by several orders of magnitude smaller than that by its fibre-related counterpart (as also pointed out by Pinho et al.), the last term on the RHS of Eq. (4) was neglected for the present study.

\subsection{Association of $G_{I c}^{f+}$ with strain-rate}

In order to ultimately provide $G_{I C}^{f+}(\dot{\varepsilon})$ for constitutive model calibration, the experimentally measured energy release rate had to be associated with an appropriate strain-rate measure. For a continuum damage mechanics-based (CDM) damage modelling approach, this strain-rate measure finds its representation in the experiment as the strain-rate of the material across a reference length $l_{\text {ref }}$ perpendicular to the advancing crack tip. As the thus obtained strain-rate was dependent on the initial value of this reference length, the DIC-based evaluation to 
determine $\dot{\varepsilon}$ was carried out for a range of $l_{\text {ref }}$. The relevant strain-rate value for a particular FE model is then the one corresponding to the finite elements' dimension in fibre direction (see illustration in Fig. 6). As the number of high-speed camera frames capturing the crack's propagation did not allow for any such evaluation beyond the first crack tip advancement, the determined relation $\dot{\varepsilon}\left(l_{\text {ref }}\right)$ refers to the strain-rate of $l_{\text {ref }}$ immediately prior to this initial crack propagation.

Figure 6 about here

\section{$5 \quad$ Results and discussion}

\subsection{Assessment of the dynamic tests' validity}

The validity of high-rate tests requires the influence of the specimen's inertia to be sufficiently small compared to its visco-elastic-(plastic) material response. This, in turn, means that the acting forces on both ends of the specimen, the input bar force $F_{\text {in }}$ and output bar force $F_{\text {out }}$, must have converged towards a state of reasonable dynamic equilibrium prior to the onset of crack propagation, and must remain within reasonable agreement throughout the fracture process. For the present study, convergence of $F_{\text {in }}$ and $F_{\text {out }}$ was facilitated by employing a ramp-like incident pulse covering both, the specimen's elastic deformation as well as its fracture process. A depiction of the measured signals of a representative incident pulse and the corresponding specimen response is provided in Fig. 7 (time axes were shifted to common arbitrary origin).

Figure 7 about here 
The state of force disequilibrium $\Delta_{F}(t)$ was quantified similar to [27]:

$$
\Delta_{F}(t)=\left|\frac{F_{\text {in }}(t)-F_{\text {out }}(t)}{\frac{1}{2}\left(F_{\text {in }}(t)+F_{\text {out }}(t)\right)}\right| \cdot 100
$$

A representative depiction of $F_{\text {in }}(t), F_{\text {out }}(t)$, and the corresponding plots of their arithmetic mean and state of force disequilibrium $\Delta_{F}(t)$ are presented in Fig. 8, showing successive force convergence in the elastic deformation domain, and varying degrees of force disequilibrium in the range of approximately $5-20 \%$ over the course of crack propagation. This level of force disequilibrium during the fracture process was slightly higher than what would have been desirable; however, it was still considered low enough to allow for drawing general conclusions from the high-rate experiments. It is, however, recommended to employ a loading pulse that further reduces $\Delta_{F}(t)$ for similar experiments in the future.

Figure 8 about here

\subsection{Experimental results}

Representative force vs. opening displacement curves for both loading-rate regimes are presented in Fig. 9. The curve corresponding to quasi-static loading exhibits the well-known stepwise degradation of force level with increasing values of opening displacement. Against the background of this quasi-static curve, its high-rate counterpart exhibits (i) a considerably stiffer response for the slope corresponding to elastic deformation, (ii) a higher force level at the onset of crack propagation, (iii) an 
overall higher force level over the course of crack propagation, and (iv) a considerably lower opening displacement for comparable values of total crack lengths $a_{t o t}$. It should, however, be noted that the state of slight force disequilibrium (see Sec. 5.1) might exaggerate the degree of phenomena (i) and (ii). It is furthermore noteworthy that the specimens tested under high-rate loading failed to re-establish a state of reasonable force equilibrium beyond values of $a_{\text {tot }}$ of approximately $10 \mathrm{~mm}$, thus rendering the force/opening displacement data beyond this point unusable.

Figure 9 about here

A summary of the determined values of $G_{I c}^{f+}$ for both loading rate regimes is given in Table 1. Compared to values of $G_{I c, Q S}^{f+}$ documented in literature for the identical material system, the result obtained under quasi-static loading falls near the upper end of values determined elsewhere, see Table 2. The value for $G_{I c}^{f+}$ obtained under highrate loading conditions exhibits a salient drop compared to its quasi-static counterpart. The experimental scatter associated with the dynamic testing results was considerable.

\section{Table 1 about here}

\section{Table 2 about here}

As the governing mechanisms on micro-scale (matrix cracking, fibre/matrix debonding, fibre rupture, and fibre pull-out) involved in tensile fracture of a laminate in fibre direction resemble, to some extent, those governing the pull-out process of z-pins in through-the-thickness-reinforced laminates, it is noteworthy that the dynamic mode I 
z-pin pull-out experiments carried out by Cui et al. [28] similarly showed a pronounced decrease in their corresponding energy release rate value under high-rate loading.

In light of the state of slight force disequilibrium present over the course of crack propagation (see Sec. 5.1), the sensitivity of the obtained value for $G_{I c, \text { dyn }}^{f+}$ with respect to the measured force history was assessed by calculating $G_{I c, d y n}^{f+}$ based on each of the two force signals individually. In the case of $F_{i n}, G_{I c, d y n}^{f+}$ was found to increase by 10.2\% with respect to the value given in Table 1; the corresponding calculation based on $F_{\text {out }}$ resulted in a decrease by $6.2 \%$. These deviations from the reference value (based on the arithmetic mean of $F_{\text {in }}$ and $F_{\text {out }}$ ) were taken as evidence that the state of slight force disequilibrium over the course of crack propagation did not excessively influence the resulting value obtained for $G_{I c, d y n}^{f+}$, especially when considering the high level of experimental scatter experienced for the high-rate tests.

Finally, an attempt was made to explain the discrepancy of the results between McCarroll's [17] (see outline in Sec. 1) and the present study. To this end, the assumption of rate-insensitivity of the key parameters $k(a)$ and $C(a)$ of McCarroll's data reduction approach was examined against the background of the data generated in the context of the present work. As a simple check, both parameters were evaluated for each loading rate regime based on the curves' elastic slope in the immediate vicinity of the specimens' elastic limit. Beyond this point, a meaningful interpretation of the results of this evaluation proved difficult in the case of the high-rate tests due to severe fluctuations of the obtained values caused by the stick-slip nature of crack propagation. By the time the elastic deformation limit was reached, the value for $k$ 
under dynamic loading exhibited a decrease by approx. $17 \%$ compared to its corresponding counterpart for quasi-static loading. Similarly, the value for $C$, measured on the basis of the curves' elastic slope in the immediate vicinity of the specimens' elastic limit, showed a decrease by approx. 50\% compared to the quasistatic loading case. On the basis of these discrepancies - albeit mildly distorted due to the existing state of slight force disequilibrium - it could be argued that the ratedependent nature of the material's properties has a considerable effect on the sample's deformation behaviour, thus necessitating a data reduction approach that takes this into account (or, alternatively, that does not explicitly rely on the material's properties in the first place). McCarroll's assumption of rate-insensitivity for those two central data reduction parameters might thus explain the discrepancy in findings between [17] and the present study.

The strain-rate values $\dot{\varepsilon}\left(l_{\text {ref }}\right)$ corresponding to both loading rate regimes are presented in Fig. 10.

Figure 10 about here

\subsection{Analysis of the strain field surrounding the crack tip}

The shape, extent, and magnitude of the strain field surrounding the crack tip was analysed via DIC, employing the GOM Aramis software package. The results of this analysis are subject to two caveats: (i) As a unidirectionally-reinforced CFRP ply's stiffness in fibre direction is by approx. one order of magnitude higher than its stiffness transverse to its fibre direction, it is assumed that the strain field measured on the outer $90^{\circ}$ ply can be taken as representative of the strain field within the specimen in 
x-direction (see Fig. 11 for coordinate system); the DIC results in y-direction, however, should be considered as being strongly influenced by the deformation behaviour of the outer $90^{\circ}$ layer. (ii) The DIC analysis' results in direct vicinity of the opening crack and the crack's tip should be disregarded due to disintegration of the speckle pattern in those regions.

Selected images from both loading rate regimes showing the state of the strain field immediately before the subsequent crack propagation are presented in Fig. 11. The time stamps given for each stage refer to the time elapsed from the last image representing a purely elastic specimen deformation; the given scale is based on 'technical strain'.

The strain field surrounding the crack tip under quasi-static loading features an extensive and sharply defined high-strain zone (colour-code: red and orange). This is in salient contrast to the strain field under high-rate loading, which shows a very compact and much less defined high-strain zone. The tails of the low-strain zone (colour-code: teal), however, can reach an extent comparable to the ones encountered for the quasistatic tests.

On the basis of this analysis, it was concluded that a larger section of the length of the critical load-carrying fibres orthogonal to the crack tip is involved in the fracture process under quasi-static than for high-rate loading. This, in turn, was taken as an indication that the fracture process under high-rate loading is a more locally confined phenomenon, involving a smaller material volume.

Figure 11 about here 


\subsection{Fractography}

The specimens' fracture surface morphology was investigated employing scanning electron microscopy (SEM) in order to assess the effect of the two loading rate regimes on the governing failure mechanisms acting on micro-scale. A $3 \mathrm{~nm}$ thick palladium/gold coating was applied prior to scanning.

Representative images for each loading rate regime showing multiple $0^{\circ}$ layers across the specimens' through-thickness direction are presented in Fig. 12. No marked differences between the respective fracture surface morphologies were detectable. In particular, the overall level of the length of fibres 'protruding' from the fracture plane, which, in turn, could indicate a change in the energy dissipated associated with fibre/matrix debonding and the subsequent fibre pull-out process, did not seem to differ noticeably. This result is congruent with the outcome of McCarroll's [17] SEM analysis, even though the loading pulse profile employed in her study differed from the one generated in the present work.

Figure 12 about here

At a higher magnification level capturing the fracture surface morphology of a single $0^{\circ}$ ply, the tendency of a greater fibre length protruding from the fracture plane with increasing distance from the adjacent $90^{\circ}$ plies was found consistently in the SEM scans of specimens of both loading rate regimes, see Fig. 13. This phenomenon can be interpreted as the adjacent $90^{\circ}$ plies' increased ability to constrain the level of fibre pull-out in the $0^{\circ}$ plies with increasing proximity (e.g., see Laffan et al. (2010) [29]).

Figure 13 about here 
Even though fractography was not able to yield any direct indications regarding the micro-mechanical origin of the change of $G_{I c}^{f+}$ under high-rate loading, it should, however, be mentioned that the absence of any obvious changes in fracture surface morphology in itself does not allow the drawing of any 'final conclusions', as the energy dissipated by each failure mechanism on micro-scale is also dependent on the respective forces involved. Against this background, in the above mentioned study carried out by Cui et al. (2017) [28], the measured negative strain-rate trend of $G_{I c}^{\text {delam }}$ was linked to a decrease in frictional resistance to pull-out between the carbon fibre/epoxy z-pins and the composite laminate under high-rate loading conditions. Provided that this mechanism, observed on a 'fibre-bundle level', similarly affects the fibre pull-out of single fibres (or small groups of fibres), this decrease in frictional resistance under high-rate loading could thus provide a (partial) explanation for the strongly negative strain-rate trend found for $G_{I c}^{f+}$ in the present study.

\section{Conclusions}

The strain-energy release rate $G_{I C}^{f+}$ of a composite laminate under high-rate tensile deformation in fibre direction was determined for $I M 7 / 8552$, employing an SHB setup and subsequent data reduction based on the area method. $G_{I c}^{f+}$ was found to considerably decrease under high-rate loading conditions compared to its quasi-static counterpart.

An analysis of the strain field surrounding the crack tip employing DIC suggested that a larger material volume was involved in the fracture process under quasi-static 
compared to high-rate loading. This, in turn, could imply the possibility of a more extensive damage zone reaching deeper into the material, thus causing a higher amount of energy to be dissipated during crack propagation under quasi-static loading. No marked differences between the fracture surfaces' morphology across the tested loading rate regimes were detectable via SEM.

Further research is recommended to cover the following aspects: (i) additional postmortem analysis to investigate the extent and morphology of the damage zone within the material to confirm/refute the above hypothesis, and (ii) determination of a loading pulse profile that further decreases the level of force disequilibrium over the course of crack propagation.

The key value of the findings presented in this paper lies in the quantification of the rate-dependent nature of $G_{I c}^{f+}$ across two loading-rate regimes. The generated data, in turn, can serve as constitutive model input for the design and dimensioning process of composite structures under impact loading. Considering the strongly negative loading

rate trend found for $G_{I C}^{f+}$, not accounting for this rate-sensitivity in the design and dimensioning process might result in a composite component's higher risk of failing in an unexpected manner under impact loading in service.

\section{Acknowledgements}

The authors would like to thank Stuart Carter, Jeffrey Fullerton, Andy Bateman, Kalin Dragnevski, and Andrew Lui for the technical support. The authors would also like to 
acknowledge Rolls-Royce plc, for their continuing support through the Solid Mechanics University Technology Centre at the University of Oxford.

\section{References}

[1] C. Soutis, Carbon fiber reinforced plastics in aircraft construction, Mater. Sci. Eng. A. 412 (2005) 171-176. doi:10.1016/j.msea.2005.08.064.

[2] G. Marsh, Composites consolidate in commercial aviation, Reinf. Plast. 60 (2016) 302-305. doi:https://doi.org/10.1016/j.repl.2016.08.002.

[3] S. Heimbs, Computational methods for bird strike simulations: A review, Comput. Struct. 89 (2011) 2093-2112.

doi:https://doi.org/10.1016/j.compstruc.2011.08.007.

[4] R. Dolbeer, S. E. Wright, J. R. Weller, A. L. Anderson, M. J. Beiger, Wildlife strikes to civil aircraft in the United States, 1990-2014., 2015. doi:10.13140/RG.2.1.2370.6649.

[5] T. Schmack, T. Filipe, G. Deinzer, C. Kassapoglou, F. Walther, Experimental and numerical investigation of the strain rate-dependent compression behaviour of a carbon-epoxy structure, Compos. Struct. 189 (2018) 256-262. doi:https://doi.org/10.1016/j.compstruct.2017.11.025.

[6] H.M. Hsiao, I.M. Daniel, Strain rate behavior of composite materials, Compos. Part B Eng. 29 (1998) 521-533. doi:10.1016/S1359-8368(98)00008-0.

[7] A. Gilat, R.K. Goldberg, G.D. Roberts, Experimental study of strain-rate- 
dependent behavior of carbon/epoxy composite, Compos. Sci. Technol. 62 (2002) 1469-1476. doi:10.1016/S0266-3538(02)00100-8.

[8] H. Koerber, J. Xavier, P.P. Camanho, High strain rate characterisation of unidirectional carbon-epoxy IM7-8552 in transverse compression and in-plane shear using digital image correlation, Mech. Mater. 42 (2010) 1004-1019. doi:http://dx.doi.org/10.1016/j.mechmat.2010.09.003.

[9] J.D. Schaefer, B.T. Werner, I.M. Daniel, Strain-Rate-Dependent Failure of a Toughened Matrix Composite, Exp. Mech. 54 (2014) 1111-1120. doi:10.1007/s11340-014-9876-0.

[10] H. Cui, D. Thomson, A. Pellegrino, J. Wiegand, N. Petrinic, Effect of strain rate and fibre rotation on the in-plane shear response of $\pm 45^{\circ}$ laminates in tension and compression tests, Compos. Sci. Technol. 135 (2016) 106-115. doi:https://doi.org/10.1016/j.compscitech.2016.09.016.

[11] M. Ploeckl, P. Kuhn, J. Grosser, M. Wolfahrt, H. Koerber, A dynamic test methodology for analyzing the strain-rate effect on the longitudinal compressive behavior of fiber-reinforced composites, Compos. Struct. 180 (2017) 429-438. doi:https://doi.org/10.1016/j.compstruct.2017.08.048.

[12] G.C. Jacob, J.M. Starbuck, J.F. Fellers, S. Simunovic, R.G. Boeman, The effect of loading rate on the fracture toughness of fiber reinforced polymer composites, J. Appl. Polym. Sci. 96 (2005) 899-904. doi:10.1002/app.21535.

[13] M. May, Measuring the rate-dependent mode I fracture toughness of 
composites - A review, Compos. Part A Appl. Sci. Manuf. 81 (2016) 1-12. doi:10.1016/j.compositesa.2015.10.033.

[14] Y. Nikishkov, G. Seon, A. Makeev, B. Shonkwiler, In-situ measurements of fracture toughness properties in composite laminates, Mater. Des. 94 (2016) 303-313. doi:https://doi.org/10.1016/j.matdes.2016.01.008.

[15] M.J. Laffan, S.T. Pinho, P. Robinson, A.J. McMillan, Translaminar fracture toughness: The critical notch tip radius of $0^{\circ}$ plies in CFRP, Compos. Sci. Technol. 72 (2011) 97-102. doi:10.1016/j.compscitech.2011.10.006.

[16] P. Kuhn, G. Catalanotti, J. Xavier, P.P. Camanho, H. Koerber, Fracture toughness and crack resistance curves for fiber compressive failure mode in polymer composites under high rate loading, Compos. Struct. 182 (2017) 164-175. doi:https://doi.org/10.1016/j.compstruct.2017.09.040.

[17] C.A. McCarroll, High Rate Fracture Toughness Measurement of Laminated Composites, PhD thesis, Imperial College London, 2011.

[18] S.T. Pinho, P. Robinson, L. Iannucci, Fracture toughness of the tensile and compressive fibre failure modes in laminated composites, Compos. Sci. Technol. 66 (2006) 2069-2079. doi:http://dx.doi.org/10.1016/j.compscitech.2005.12.023.

[19] Hexcel product data sheet for HexPly 8552, (2016). http://www.hexcel.com/user_area/content_media/raw/HexPly_8552_eu_Data Sheet.pdf.

[20] R. Gerlach, C. Kettenbeil, N. Petrinic, A new split Hopkinson tensile bar design, 
Int. J. Impact Eng. 50 (2012) 63-67.

doi:http://dx.doi.org/10.1016/j.ijimpeng.2012.08.004.

[21] ASTM E399-12e3, Standard Test Method for Linear-Elastic Plane-Strain Fracture Toughness KIc of Metallic Materials, ASTM International, West Conshohocken, PA, (2012).

[22] G. Catalanotti, P.P. Camanho, J. Xavier, C.G. Dávila, A.T. Marques, Measurement of resistance curves in the longitudinal failure of composites using digital image correlation, Compos. Sci. Technol. 70 (2010) 1986-1993.

[23] H. Zhao, Material behaviour characterisation using SHPB techniques, tests and simulations, Comput. Struct. 81 (2003) 1301-1310.

doi:https://doi.org/10.1016/S0045-7949(03)00044-0.

[24] W.F. Hosford, Mechanical behavior of materials, 2nd ed., Cambridge University Press, Cambridge, New York, Melbourne, Madrid, Cape Town, Singapore, Sao Paulo, Delhi, Dubai, Tokyo, 2010.

[25] N. Sela, O. Ishai, Interlaminar fracture toughness and toughening of laminated composite materials: a review, Composites. 20 (1989) 423-435. doi:http://dx.doi.org/10.1016/0010-4361(89)90211-5.

[26] M.J. Laffan, S.T. Pinho, P. Robinson, L. Iannucci, Measurement of the in situ ply fracture toughness associated with mode I fibre tensile failure in FRP. Part I: Data reduction, Compos. Sci. Technol. 70 (2010) 606-613. doi:10.1016/j.compscitech.2009.12.016. 
[27] G. Ravichandran, G. Subhash, Critical Appraisal of Limiting Strain Rates for Compression Testing of Ceramics in a Split Hopkinson Pressure Bar, J. Am. Ceram. Soc. 77 (1994) 263-267. doi:10.1111/j.1151-2916.1994.tb06987.x.

[28] H. Cui, M. Yasaee, G. Kalwak, A. Pellegrino, I.K. Partridge, S.R. Hallett, G. Allegri, N. Petrinic, Bridging mechanisms of through-thickness reinforcement in dynamic mode I\&II delamination, Compos. Part A Appl. Sci. Manuf. 99 (2017) 198-207. doi:https://doi.org/10.1016/j.compositesa.2017.04.009.

[29] M.J. Laffan, S.T. Pinho, P. Robinson, L. Iannucci, Measurement of the in situ ply fracture toughness associated with mode I fibre tensile failure in FRP. Part II: Size and lay-up effects, Compos. Sci. Technol. 70 (2010) 614-621. doi:10.1016/j.compscitech.2009.12.011.

[30] P.P. Camanho, P. Maimí, C.G. Dávila, Prediction of size effects in notched laminates using continuum damage mechanics, Compos. Sci. Technol. 67 (2007) 2715-2727. doi:https://doi.org/10.1016/j.compscitech.2007.02.005.

[31] G. Catalanotti, A. Arteiro, M. Hayati, P.P. Camanho, Determination of the mode I crack resistance curve of polymer composites using the size-effect law, Eng. Fract. Mech. 118 (2014) 49-65. doi:https://doi.org/10.1016/j.engfracmech.2013.10.021. 
Figures

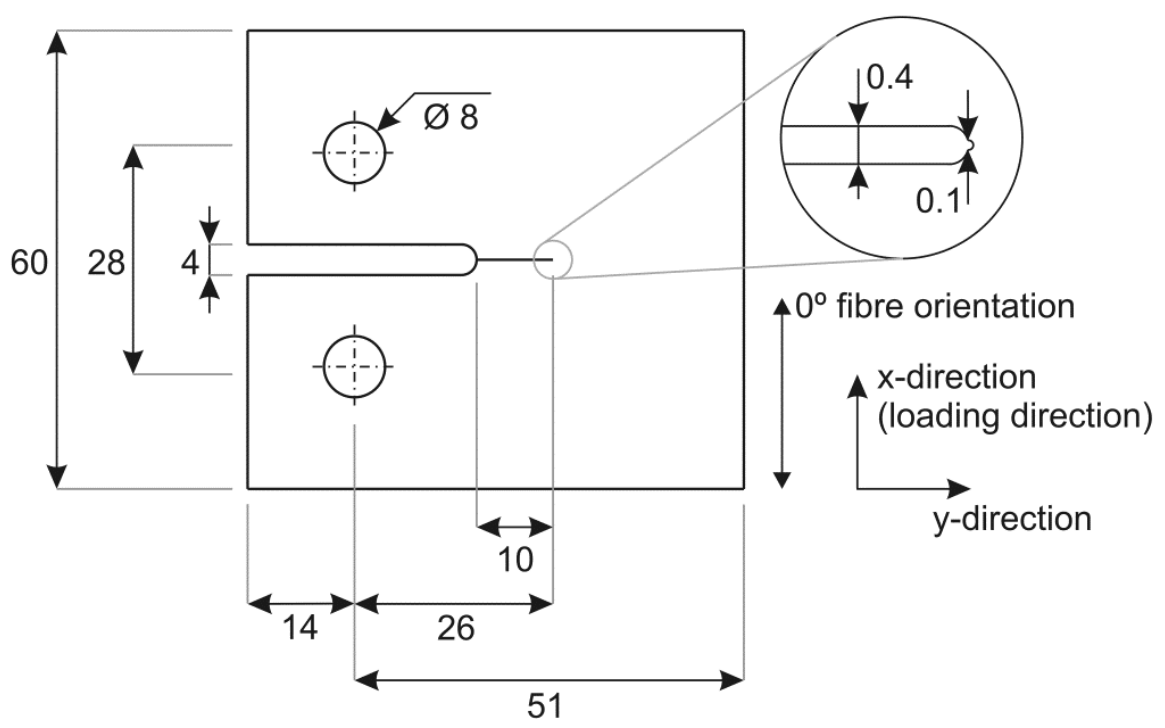

Fig. 1. Geometry of the CT specimen (dimensions in $\mathrm{mm}$ ) 1200dpi; grayscale; width: $140 \mathrm{~mm}$
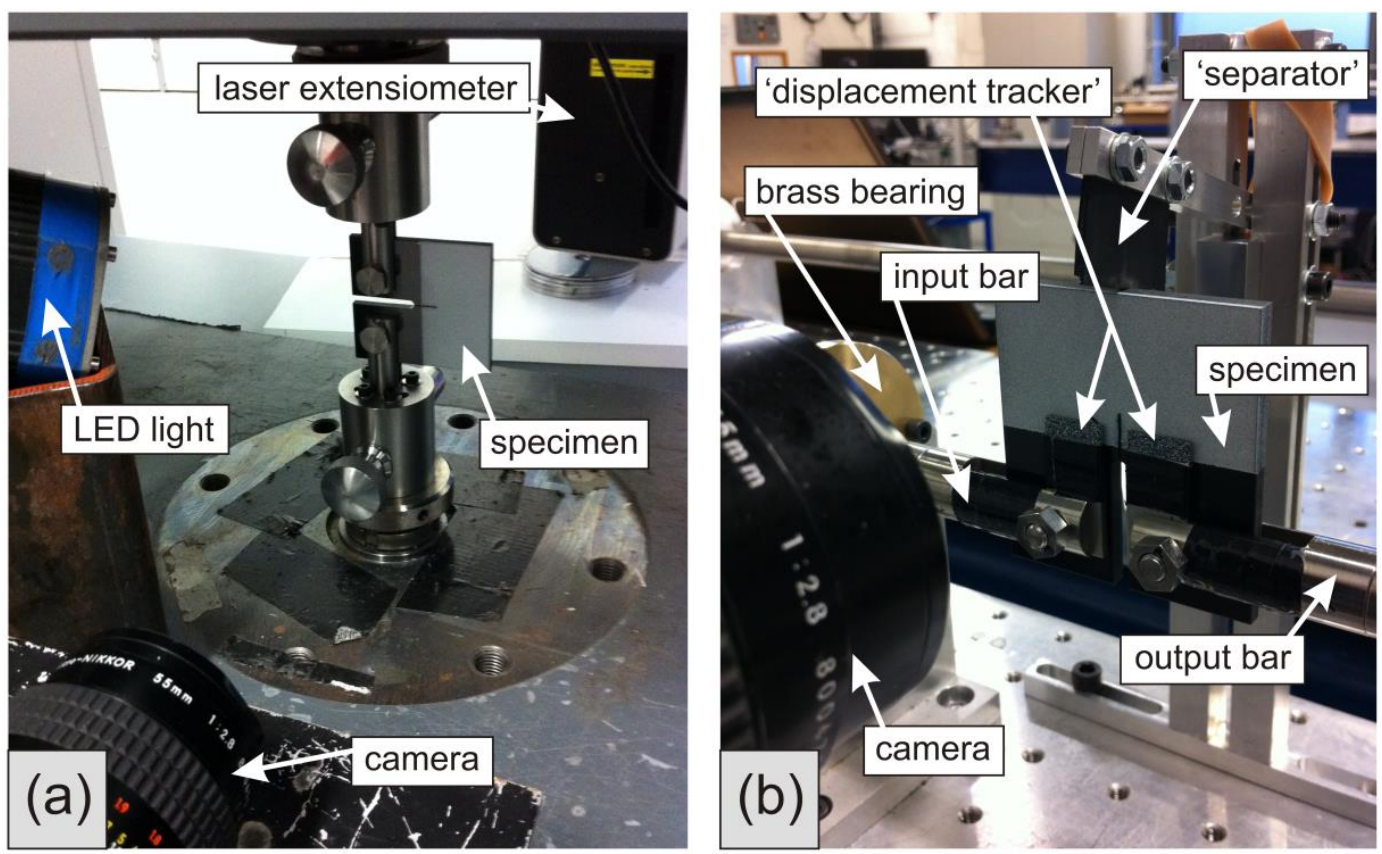
Fig. 2. Experimental setup for the tests under (a) quasi-static, and (b) high-rate loading conditions $600 \mathrm{dpi}$; colour; width: $140 \mathrm{~mm}$

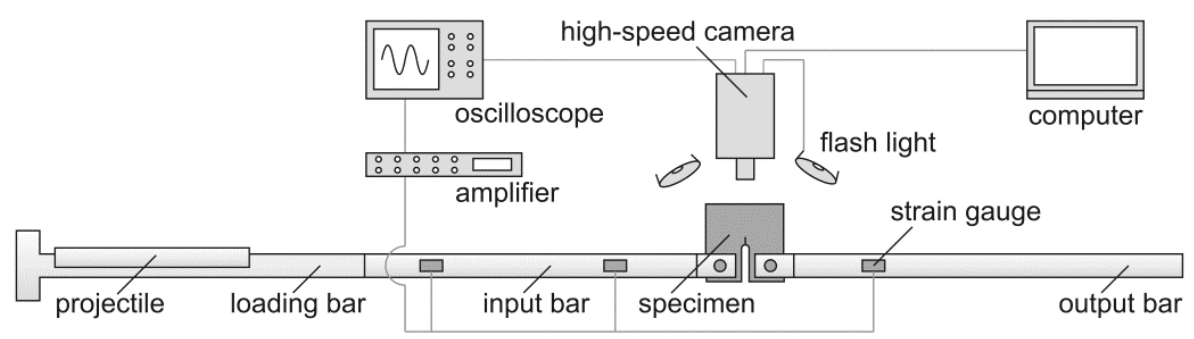

Fig.3. Split-Hopkinson tension bar setup and data acquisition for the high-rate experiments $1200 \mathrm{dpi}$; grayscale; width: $190 \mathrm{~mm}$

(a)
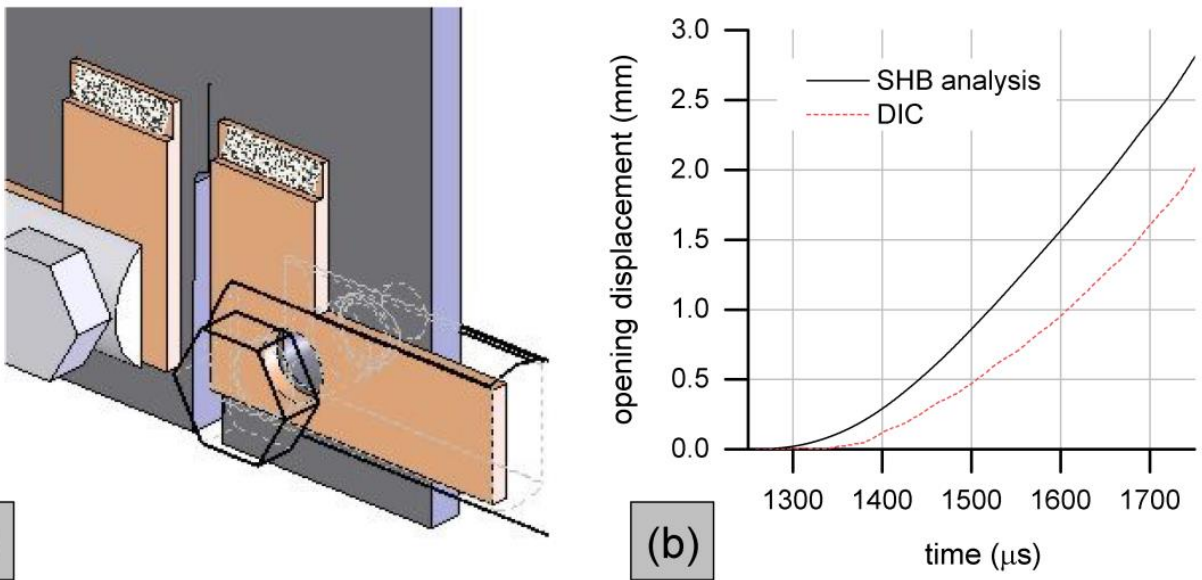

Fig. 4. (a) 'displacement tracker' to allow for a DIC-based determination of the opening displacement; (b) comparison between an opening displacement measurement based on the SHB analysis and a DIC-based approach 600dpi; colour; width: $190 \mathrm{~mm}$ 


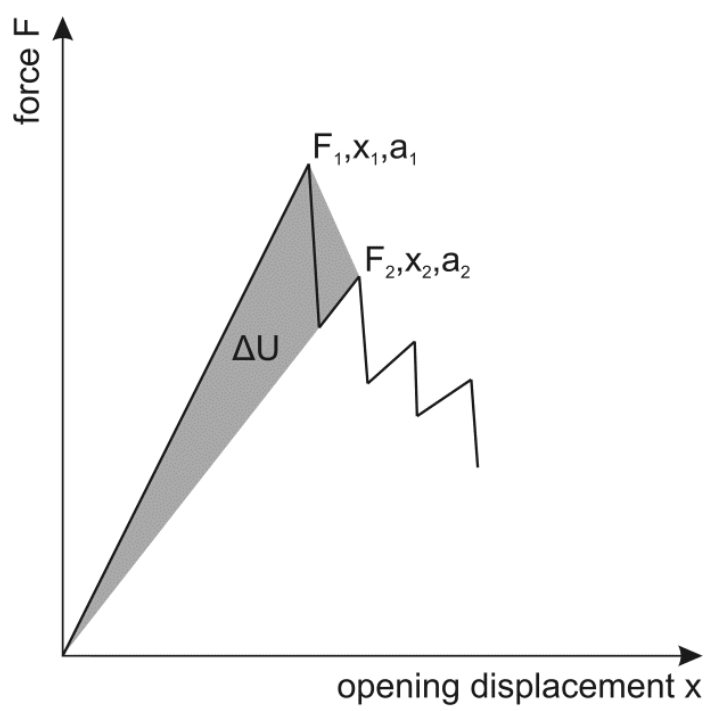

Fig. 5. Determination of $G_{I c}^{\text {lam }}$ based on the area method 1200dpi; grayscale; width: $90 \mathrm{~mm}$

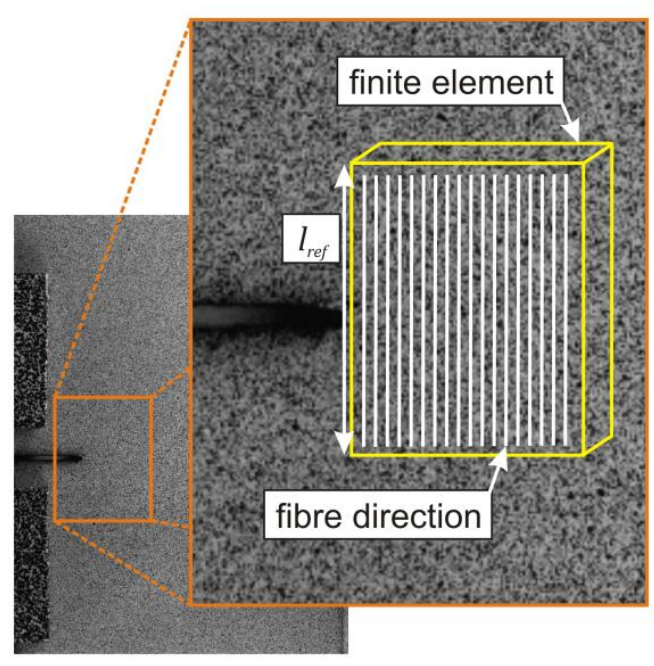

Fig. 6. Determination of strain-rate with its relation to a CDM-based modelling approach 600dpi; colour; width: $90 \mathrm{~mm}$ 


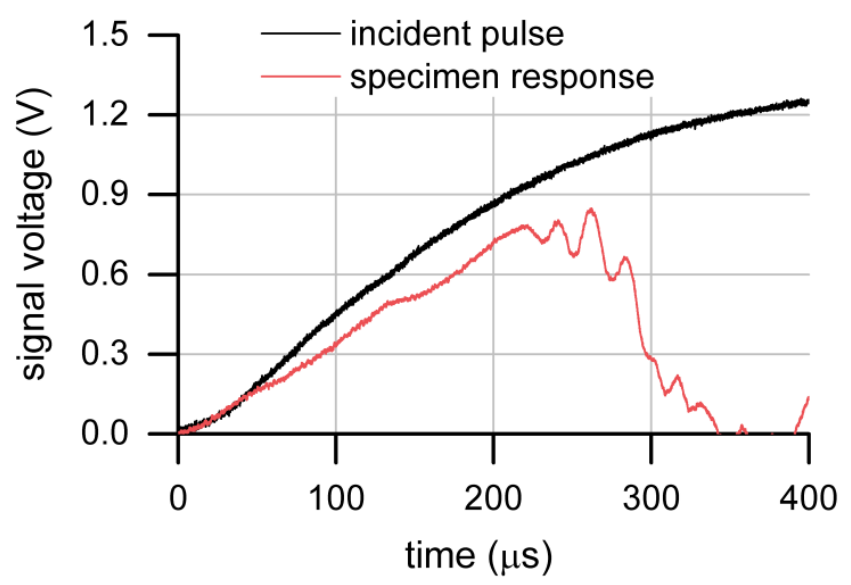

Fig. 7. Incident pulse and corresponding specimen response $F_{\text {out }} 1200 \mathrm{dpi}$; colour; width: $90 \mathrm{~mm}$

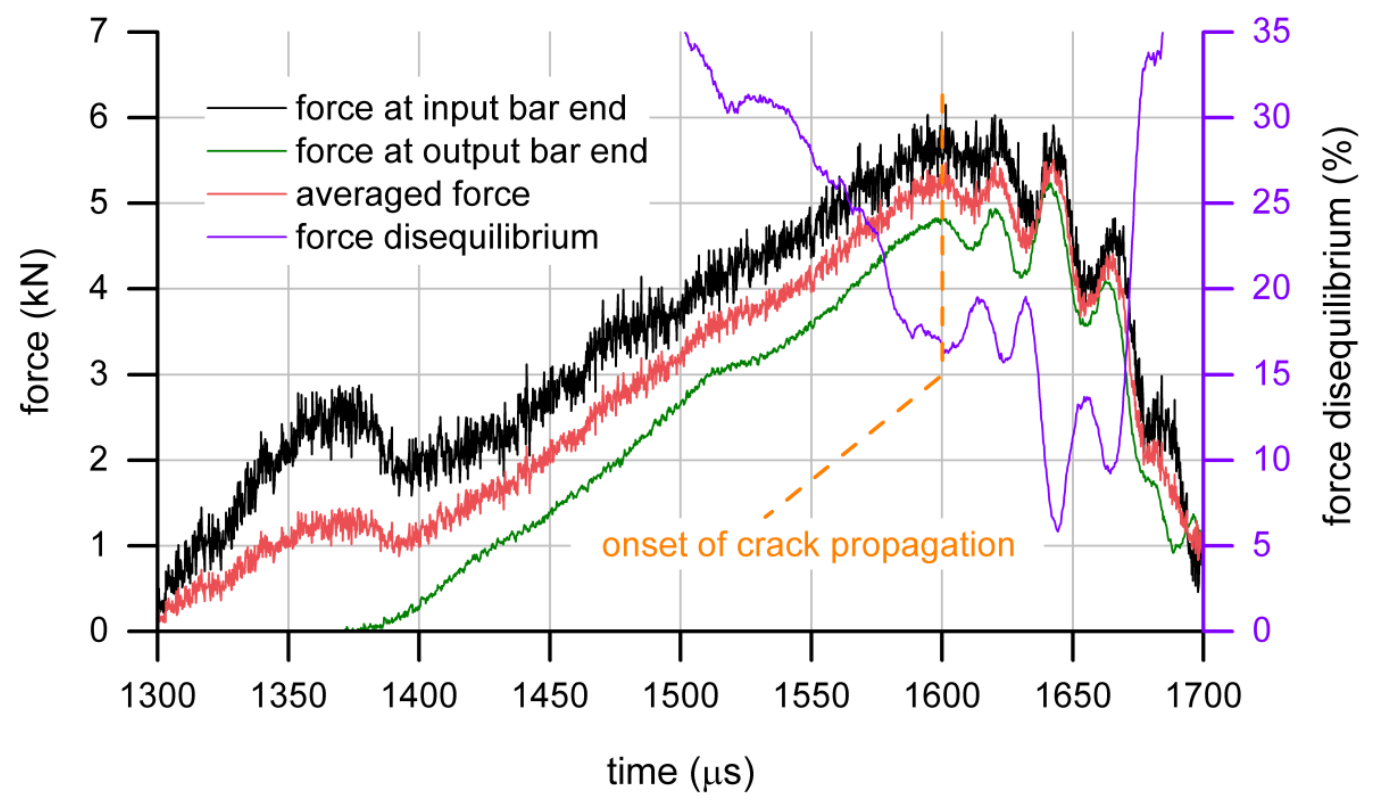

Fig. 8. Force convergence towards dynamic equilibrium $1200 d p i$; colour; width:

$140 \mathrm{~mm}$ 


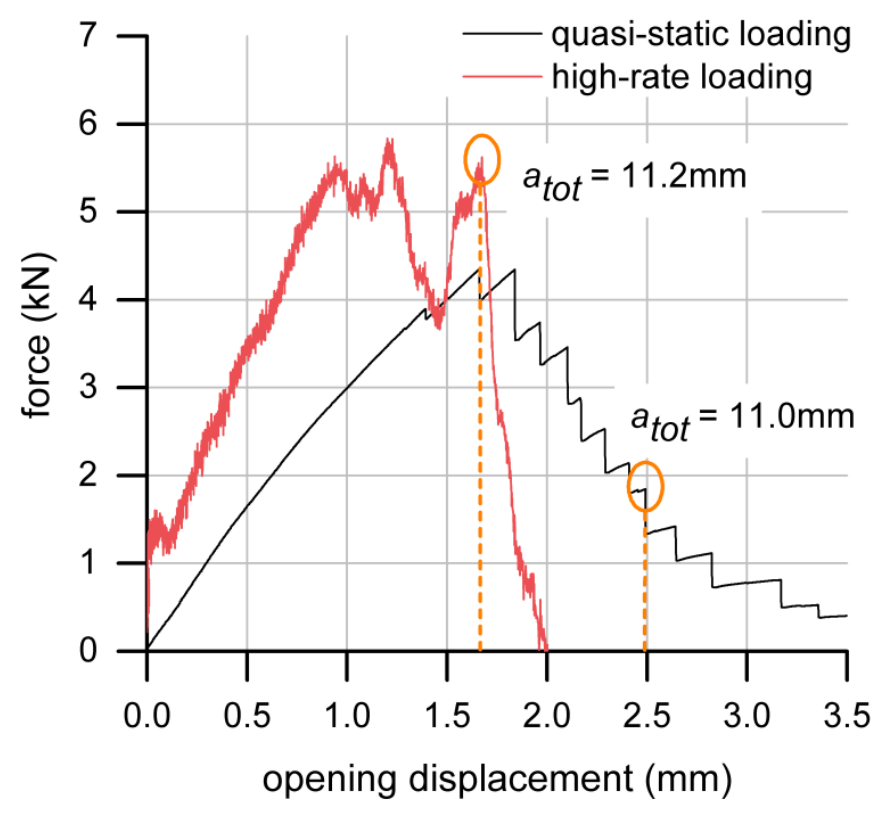

Fig. 9. Representative force vs. opening displacement curves for both loading-rate regimes $1200 \mathrm{dpi}$; colour; width: $90 \mathrm{~mm}$
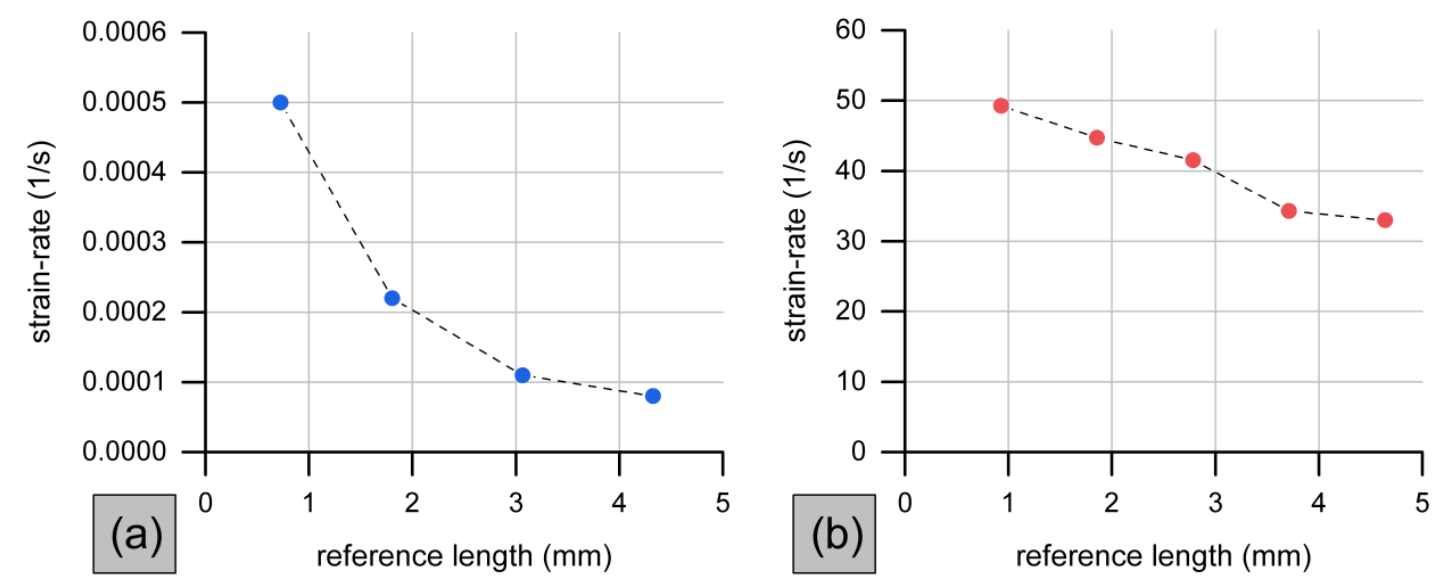

Fig. 10. Relation of reference length to determined strain-rate for (a) quasi-static loading, and (b) high-rate loading 1200dpi; colour; width: $190 \mathrm{~mm}$ 

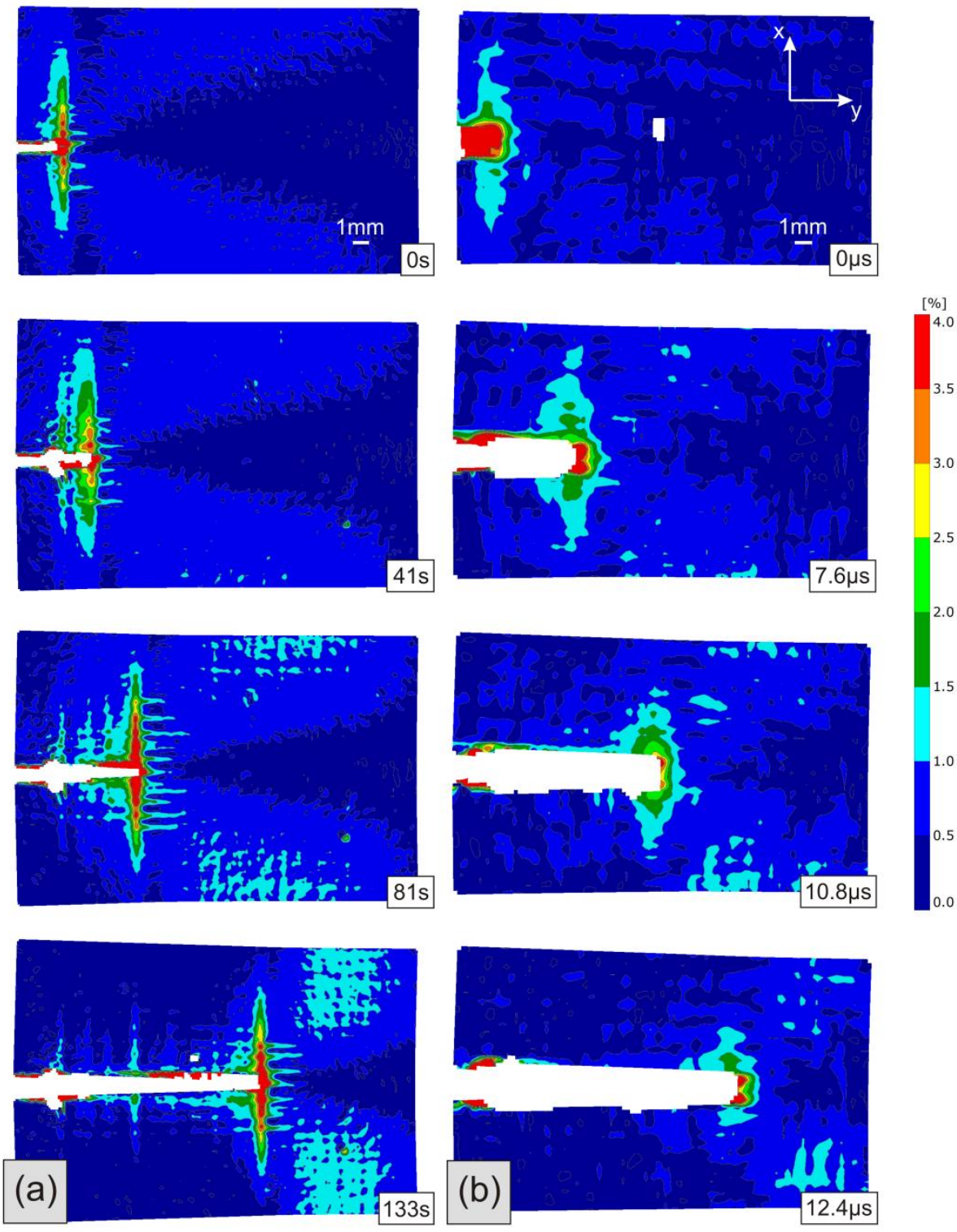

Fig. 11. Strain field surrounding the crack tip for several stages of crack tip advancement under (a) quasi-static, and (b) high-rate loading $600 \mathrm{dpi}$; colour; width: $190 \mathrm{~mm}$ 


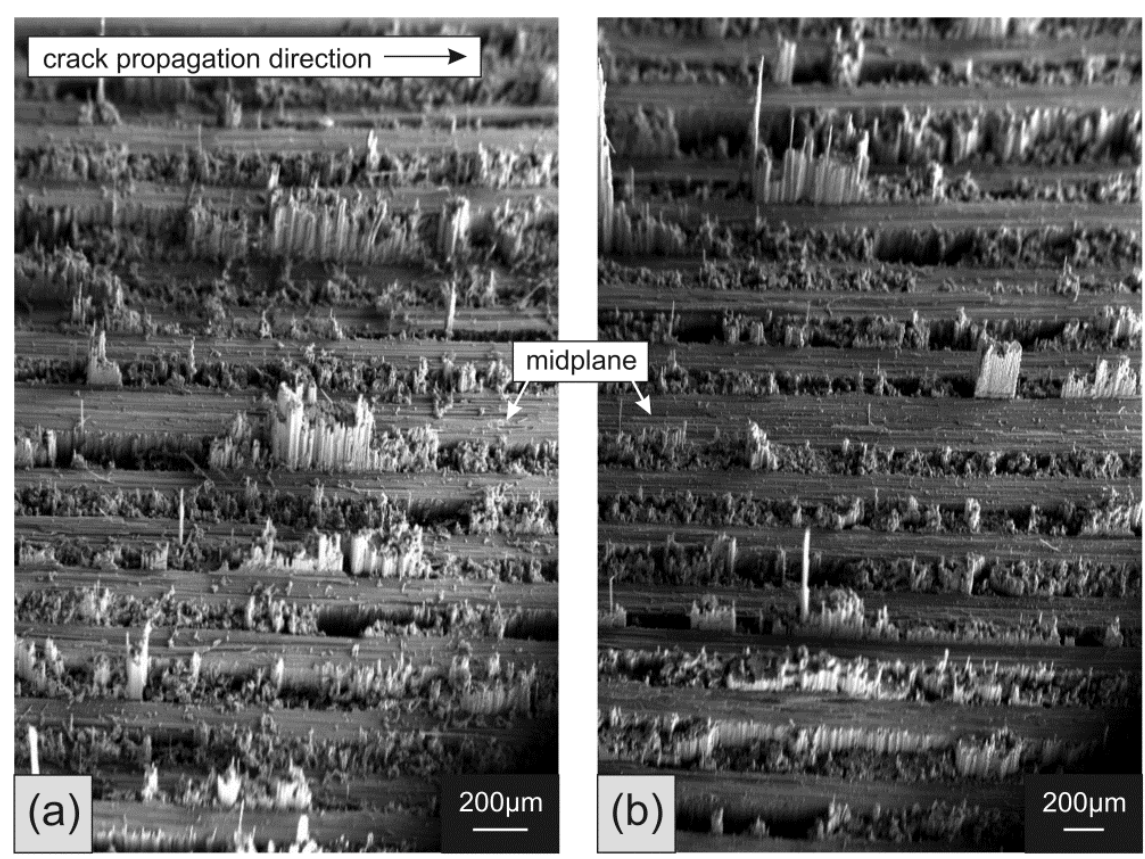

Fig. 12. SEM images of multiple $0^{\circ}$ plies in through-thickness direction for (a) quasistatic loading, and (b) high-rate loading 600dpi; grayscale; width: $190 \mathrm{~mm}$
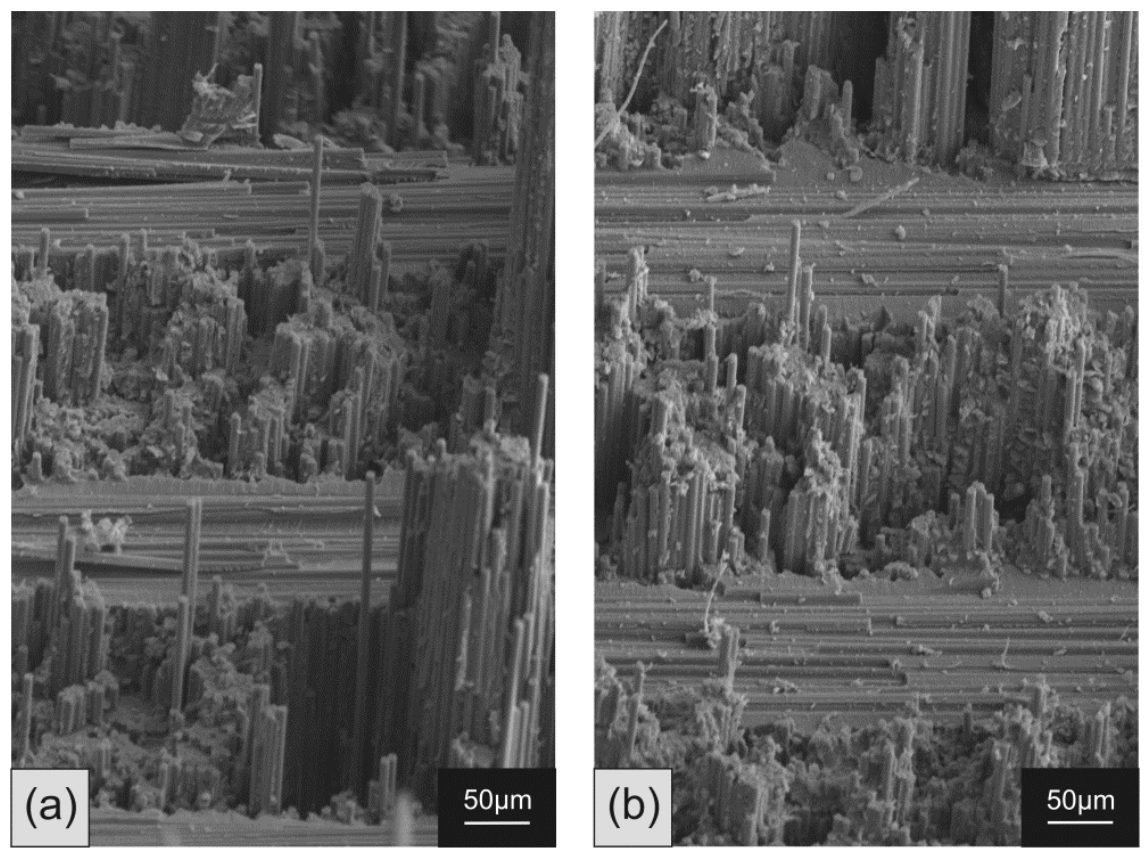
Fig. 13. SEM images of a single $0^{\circ}$ ply showing the constraining effect of $90^{\circ}$ layers on adjacent $0^{\circ}$ plies for (a) quasi-static loading, and (b) high-rate loading 600dpi; grayscale; width: $190 \mathrm{~mm}$ 


\section{Tables}

Table 1: Summary of the determined energy release rate values for both loading rate regimes

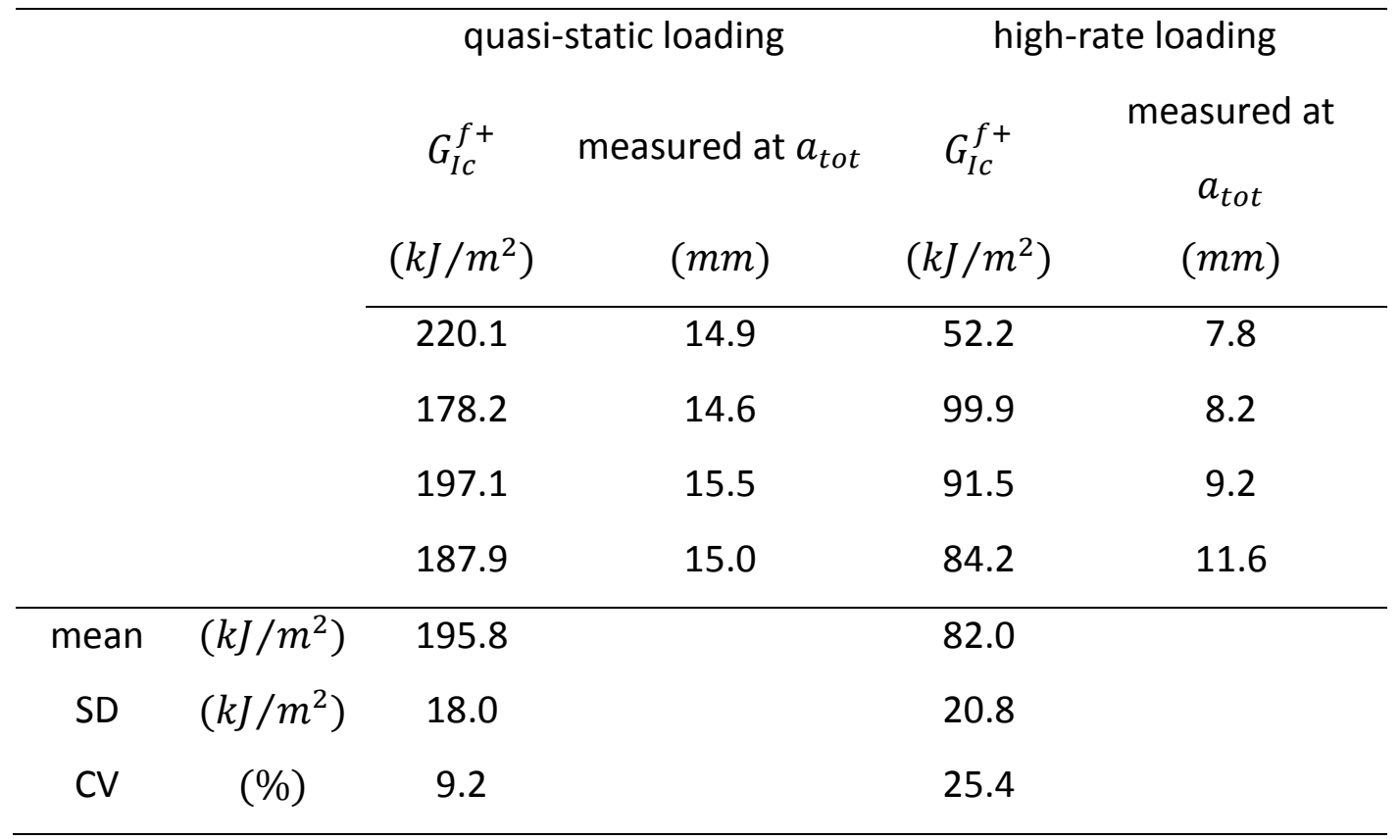

Table 2: $G_{I C, Q S}^{f+}$ for $I M 7 / 8552$ documented in literature

\begin{tabular}{ccc}
\hline & $\begin{array}{c}\text { mean prop. value } \\
\left(\mathrm{kJ} / \mathrm{m}^{2}\right)\end{array}$ & $\begin{array}{c}\mathrm{CV} \\
(\%)\end{array}$ \\
\hline Camanho et al. (2007) [30] & 81.5 & 7.6 \\
Catalanotti et al. (2010) [22] & 133.7 & - \\
Laffan et al. (2011) [15] & 147.2 & 8.2 \\
McCarroll (2011) [17] & 74.9 & 9.1 \\
Catalanotti et al. (2014) [31] & 205 & - \\
\hline
\end{tabular}




\title{
Determination of the strain-energy
}

release rate of a composite laminate

under high-rate tensile deformation in

fibre direction

\author{
Hoffmann, Justus
}

Elsevier

Hoffmann J, Cui $\mathrm{H}$, Petrinic N, Determination of the strain-energy release rate of a composite laminate under high-rate tensile deformation in fibre direction, Composites Science and Technology, Volume 164, August 2018, pp. 110-119 http://dx.doi.org/10.1016/j.compscitech.2018.05.034 Downloaded from Cranfield Library Services E-Repository 